\title{
Egresados de nuevas carreras universitarias: Competir desigualmente en el mercado de trabajo
}

\section{Graduates of New University Careers: Unequal Competition on the Labour Market}

\author{
Javier Damián Simón \\ Universidad del Papaloapan, Oaxaca, México.
}

Recibido: $14-10-16$

Aprobado: 29-03-17

\section{Correspondencia}

Email: damian_ce@hotmail.com

\section{Citar como:}

Simón J. (2017). Egresados de nuevas carreras universitarias: competir desigualmente en el mercado de trabajo. Propósitos $y$ Representaciones, 5(1), 129-203 doi: http:// dx.doi.org/10.20511/pyr2017.v5n1.146

(C) Universidad San Ignacio de Loyola, Vicerrectorado de Investigación y Desarrollo, 2017. (cc)BY-NC-ND Este artículo se distribuye bajo licencia CC BY-NC-ND 4.0 Internacional (http://creativecommons.org/licenses/by-nc-nd/4.0/). 


\section{Resumen}

El objetivo del trabajo es describir el proceso de inserción laboral en un contexto de mercado de trabajo local de los egresados para identificar rasgos de discriminación originados por su perfil profesional híbrido ${ }^{1}$; mediante un estudio de caso y un enfoque cualitativo se investigó a los egresados de las dos primeras generaciones de la Licenciatura en Ciencias Empresariales de una pequeña universidad pública mexicana. Los resultados contradicen las ventajas atribuidas a las carreras universitarias híbridas, pues lejos de facilitar la incorporación al trabajo se encontró que los egresados híbridos compiten de manera desigual por las ofertas de trabajo debido a dos situaciones: la falta de conocimiento social de su profesión por el conjunto de empleadores que origina la ausencia de demanda de dichos profesionistas y, la falta de identidad profesional de los egresados que ocasiona que no sean capaces de justificar y defender la utilidad de su formación híbrida.

Palabras clave: Educación superior, universidad, egresados, educación y empleo, inserción laboral.

\section{Summary}

The purpose of the study is to describe the process of job insertion in a context of the local labour market of the graduates to identify traits of discrimination originated by their hybrid professional profile ${ }^{2}$. A case study and a qualitative approach were used to investigate the graduates of the first two generations of the Business Sciences Degree of a small Mexican public university. The results contradict the advantages attributed to hybrid university careers, far from facilitating the incorporation to the work, it found that hybrid graduates compete of unequal way for job offers due to two situations: lack of social knowledge of their profession by all employers that originates the absence of demand of these professionals, and lack of professional identity of graduates

1 En la educación superior mexicana se observan tendencias de especialidades transversales que aluden a la polifuncionalidad y la multidisciplinariedad, mismas que han dado origen a una oferta de carreras universitarias "nuevas". A lo largo de este trabajo de acuerdo a los especialistas y sin afán de otorgarle un sentido peyorativo, se les denomina carreras híbridas. 
that causes they are unable to justify and defend the usefulness of their hybrid training.

Keywords: Higher education, University, Graduates, employment, education, job insertion. 


\section{Introducción}

\section{Contextualización y problematización del tema.}

Aludiendo a nuevas exigencias del mercado de trabajo según diagnósticos hechos por organismos internacionales y atendiendo a las recomendaciones de los responsables de la planeación educativa nacional, el sistema de educación superior mexicano ha emprendido acciones como la revisión de las currícula, la adecuación de los perfiles profesionales, la creación y oferta de carreras universitarias que mezclan dos o más disciplinas tradicionales dando lugar a las carreras híbridas (DGEST, 2009; CUP, 2009; Barrón y Gómez, 2004). A modo de ejemplo, a partir del año 2009 el subsistema del Tecnológico Nacional de México oferta la "Ingeniería en Gestión Empresarial", las Universidades Tecnológicas la "Ingeniería en Desarrollo e Innovación Empresarial” y la "Ingeniería en Administración", en tanto las Universidades Politécnicas lo hacen con la "Ingeniería Financiera y Gestión de Proyectos"; en los tres casos anteriores estas nuevas opciones educativas sustituyen a la tradicional y conocida Licenciatura en Administración de Empresas. La justificación para lo anterior es que la formación profesional híbrida refuncionaliza a las profesiones hacia esquemas de mayor flexibilidad y permite a los graduados incorporarse al trabajo con mayores ventajas y facilidades pues ésta responde a las exigencias del mercado de trabajo actual caracterizado por ser polivalente, multidisciplinario e interdisciplinario (Dahrendorf, 1986; Psacharopoulos, 2005).

Los escasos estudios efectuados a la fecha en México sobre graduados universitarios híbridos han encontrado dos problemas: el escaso reconocimiento social ${ }^{3}$ de la profesión originado por el desconocimiento del perfil del graduado híbrido y, la ausencia de identidad profesional entre

3 La literatura especializada alude a la categoría "reconocimiento social" de la profesión, misma que para obtenerla se requiere que transcurra cierto tiempo una vez llevada a cabo la inserción laboral y poder evaluar el grado de contribución de la profesión a las necesidades de la sociedad, en el caso que nos ocupa debido a que el periodo de tiempo que ha transcurrido es muy corto se utiliza en todo el texto la expresión "conocimiento social" de la profesión. 
los individuos que ostentan dicha (Jiménez, 2009; Damián, 2015). Esta problemática representa un campo emergente en la investigación educativa pues poco se conoce de ella y por tanto abre grandes posibilidades para generar conocimiento que responda a las nuevas interrogantes sobre los procesos de desarrollo profesional, así como de las situaciones sociales y laborales que viven este tipo de egresados al acceder al mercado de trabajo $\mathrm{y}$, en caso de ser necesario señalar o evidenciar los desatinos de ofertar este tipo de profesiones. De las situaciones anteriores se derivó la necesidad de efectuar un estudio sobre las condiciones en las que tiene que competir para su inserción laboral el egresado con formación híbrida de la Licenciatura en Ciencias Empresariales (LCE), frente a los egresados de carreras administrativas muy conocidas por el conjunto de empleadores, pues desde su experiencia enfrenta un mercado laboral discriminatorio y con desventajas ante los egresados de carreras tradicionales. A fin de que el lector se situé en el contexto de la problemática estudiada, a continuación se describe un poco más dicha situación.

La LCE inició actividades en octubre del año 2005 y para el año escolar 2016 ha recibido a once generaciones de las cuales ya egresaron las primeras siete. Según pláticas informales con algunos egresados, éstos mencionaron que en su búsqueda de empleo, las empresas o los empleadores carecen de información sobre el campo laboral de la profesión y desconocen la figura del LCE, situación que desde su propio imaginario origina que tengan que competir por los puestos de trabajo de manera desigual con los profesionistas de áreas afines como los administradores y contadores, pues estas carreras tradicionales gozan del reconocimiento social de parte de los empleadores; es decir, consideran que están enfrentando un mercado laboral discriminatorio al competir de manera desigual en la arena profesional. Abordar este tema emergente de investigación resulta importante pues contribuirá en la generación de información a partir de la investigación empírica, para conocer la situación de discriminación que enfrentan los egresados con formación universitaria híbrida durante el proceso de inserción laboral en 
una región escasamente industrializada del estado de Oaxaca. Son dos los objetivos que persiguen las Instituciones de Educación Superior (IES), al ofertar programas educativos híbridos: 1) responder a las exigencias actuales del mercado de trabajo formando profesionales flexibles y polivalentes y, 2) incrementar las oportunidades de trabajo a los egresados híbridos en contraste con los de carreras tradicionales (Damián, 2015a); de allí el interés de efectuar un estudio que permitiera evaluar en la LCE los dos objetivos básicos anteriores y la congruencia entre el currículo universitario híbrido y las nuevas exigencias del mercado laboral (Barrón y Gómez, 2004). Por todo lo anterior, el objetivo principal de la investigación fue describir el proceso de inserción laboral en un contexto de mercado de trabajo local de los egresados de la LCE, a fin de identificar rasgos de discriminación originados por su perfil profesional híbrido.

\section{Marco teórico y conceptual.}

\section{Antecedentes de las profesiones universitarias híbridas.}

Se afirma que es necesario reestructurar la oferta de educación superior por tres razones: 1) mayor flexibilidad, adaptabilidad y pertinencia de la formación profesional para responder al contexto ocupacional actual caracterizado por cambios rápidos en los requerimientos de calificación laboral; 2) la imprevisibilidad, indeterminación, adaptabilidad, capacidad de aprendizaje y formación continua, caracterizan al mundo del trabajo y a los cuales la educación debe responder (Muñoz y Rodríguez 2004; Barrón 2005) y; 3) el surgimiento de nuevas formas y tendencias de organización, diferenciación, identidad y delimitación de generación y apropiación del conocimiento. Muchas profesiones tradicionales caracterizadas por claras definiciones, delimitaciones e identidades de su área, están sometidas a tensiones y cuestionamientos derivadas de la emergencia de nuevos campos híbridos o transdisciplinarios que ofrecen nuevas composiciones, demarcaciones, límites e identidades entre diversos saberes, campo fértil para la oferta de carreras universitarias híbridas -que en teoría- forman a los 
recursos humanos que responden a las exigencias de la nueva organización del trabajo (Universia, 2009; DGEST, 2009; CUP, 2009).

\section{Las profesiones universitarias híbridas: finalidades y características.}

Las ocupaciones se clasifican en tres grandes grupos: 1) ocupaciones nuevas, que no existían hace algunos años, 2) ocupaciones en transformación que aunque mantienen su nombre tradicional se han transformado incorporando nuevos contenidos y funciones y, 3) ocupaciones emergentes que representan respuestas a las necesidades emergentes de las personas y las empresas. Dentro de las ocupaciones en transformación se incluyen a los profesionales híbridos, que tienen una formación en dos o más campos de conocimiento y la utilizan para ejercer una ocupación que se desarrolla en la frontera entre dos o más disciplinas (Damián, 2014). Para Jiménez (2009a), una profesión híbrida se caracteriza por que su "plan de estudios se diseñó a partir de la convergencia de dos disciplinas reconocidas", lo que lleva implícito que cada una de las disciplinas que se mezclan para dar paso a la hibridación poseen una clara y específica delimitación de su campo de actuación; de igual manera utiliza el término de profesión interdisciplinaria para explicitar que es una profesión en la que los "conceptos y métodos de dos disciplinas se articulan y subsumen en un objeto común y en una problemática determinada” (p.59).

Holguín (2011), menciona que la formación universitaria híbrida al romper el paradigma de la especialización proporciona una oportunidad doble al egresado pues en caso de emplearse en una empresa, por su formación híbrida puede desempeñarse en más de dos áreas, es decir, puede concursar por varias opciones laborales. En contraparte Paéz (2009), señala que los egresados universitarios híbridos se encuentran en una zona de ambigüedad profesional pues se mueven laboralmente entre las fronteras de las disciplinas que articulan sin una delimitación marcada de su actuación profesional; en relación a lo anterior Damián (2015), encontró que éstos generalmente tienden a "invadir" el campo de actuación de los profesionistas tradicionales o bien se convierten en el "comodín" de las empresas, es decir, se les asignan 
actividades de naturaleza administrativa que no exigen conocimientos especializados. Sáez (2007), menciona tres características del profesionista híbrido: 1) su campo de actuación profesional es más difícil que el del profesionista especialista pues en sus inicios no sabe que conocimientos o trabajos cubre ni con que intensidad; 2) el profesionista especialista elige un campo de conocimientos y se limita a la complejidad técnica del mismo, pero el profesional híbrido tiene una misión más amplia ya que su participación será para solución de problemas importantes que requieran de enfoques más complejos y multidisciplinarios y; 3) el profesional híbrido no deja de ser especialista en algo, pero tiene que ser menos especialista que los muchos especialistas, aunque lo suficiente como para tener un diálogo fluido y operativo con los especialistas. Para el profesionista híbrido no existe un punto determinado entre el mucho de nada o poco de mucho, es decir, no tiene una delimitación clara de su actuación profesional ${ }^{4}$ (Jiménez, 2009b; Paéz, 2009).

\section{Identidad profesional y reconocimiento social en los profesionistas}

\section{híbridos.}

Bajo una forma individual y un entendimiento subjetivo de la carrera profesional resulta importante preguntarse cómo los individuos dan sentido a sus carreras, a sus historias personales y a las capacidades, actitudes y creencias que han adquirido (Arnold \& Jackson, 1997; como se citó en Jenschke, 2004); lo anterior reviste importancia pues uno de los factores que contribuyen a la diligencia en la búsqueda del primer empleo lo es el que el egresado tenga plena conciencia del papel que jugará en el entramado del campo profesional, de allí que algunos enfoques sugieran que durante su formación profesional los individuos no sólo deben preocuparse por adquirir capacidades propias de la carrera universitaria, sino de construir una identidad de la carrera (Miejers, 1998), además los modelos de base

4 De manera coloquial y a modo de broma algunos egresados tomando como referente a su experiencia en el desarrollo de sus actividades laborales, se autodenominan "híbridos transformer" o "mil usos". 
individual (teoría cognitivo-transaccional, teoría cognitivo-conductual y teoría de la disonancia), y los modelos integrales (Modelo Vitamina) mencionan que a la hora de analizar la inserción laboral de un individuo se relacionan aspectos individuales y contextuales mismos que juegan un papel crucial en dicho proceso.

Para Ávila y Cortés (2007, p.54), la identidad profesional es "aquella representación que se crea alrededor de un campo específico de labor el cual se reconoce en la sociedad, y entre un grupo de individuos que se identifican como miembros del mismo, cuya característica es compartir dicha representación social de la profesión y su sentido de pertenencia”. Hatum y Rivarola (2007, p.27), definen identidad profesional como el "conjunto de hábitos que una persona ha desarrollado en el ámbito de su trabajo y la potencial proyección de otros para cultivar en el futuro". Tener una identidad profesional implica pertenecer a cierto grupo, identificarse a sí mismo como profesionista en un campo de actuación específico, con un alto nivel de habilitación profesional y con respaldo y reconocimiento institucional y; supone la existencia del reconocimiento social en el imaginario colectivo existiendo una idea clara y definida de lo que implica pertenecer a esa profesión. Actualmente las formas de organización del trabajo fundamentadas en el individualismo, donde lo central es la realización personal en un contexto de fuerte competencia existe una situación de crisis identitaria permanente (Dubar, 2000). Por lo anterior se entiende la razón por la cual las profesiones híbridas carecen de un referente en el imaginario social, lo que implica una falta de claridad acerca del campo de desempeño laboral e indefinición en el perfil del sujeto como profesional, lo que contribuye a dificultar la formación de su identidad profesional. Los individuos que estudian profesiones híbridas presentan grandes dificultades para conformar su identidad profesional al menos por tres razones (Meijers 1998; Dubar 2000; Jenschke 2004):

a. No existe en el imaginario social (empleadores/sociedad) una representación de la labor del profesionista híbrido, se carece de un marco de referencia originando que el graduado asimile algún modelo de profesión que antecedió a su formación híbrida vinculando su 
práctica profesional a una carrera tradicional desvirtuando el objetivo de la formación híbrida.

b. Al no existir el marco de referencia práctico, su preparación profesional es mayoritariamente teórica, sustentada en las necesidades y exigencias que los responsables de la planeación educativa nacional "afirman" haber detectado según los diagnósticos de los mercados laborales, sin que ello implique la existencia real de dichas necesidades.

c. Los docentes poseen carreras tradicionales originado un sesgo en el proceso de formación y haciendo una interpretación indecisa del campo profesional, es decir, los alumnos que estudian una profesión híbrida carecen de un referente externo del profesional en la disciplina a la que van a dedicarse, situación grave si se tiene en cuenta que la identidad profesional empieza a gestarse desde las aulas universitarias. En el caso del programa educativo analizado no se cuenta con docentes que tengan la LCE que sirvan como un referente externo de la práctica laboral para los alumnos.

Otra variable que influye en la inserción laboral de los egresados es el reconocimiento social de la profesión, referida como "la valoración que entre los diversos estratos socioeconómicos tienen determinadas profesiones. Esto incluye tanto la valoración social de la palabra del profesional (el reconocimiento de la autoridad profesional y/o intelectual) como la aceptación de ciertas profesiones en determinados círculos sociales" (Bobadilla y Torres, 2002:98); así el reconocimiento por parte de la colectividad de la importancia del servicio prestado por los profesionales se constituye en su principal fuente de prestigio, mismo que está asociado fundamentalmente tanto a la utilidad social de la profesión como a la imposibilidad de que la ejerzan personas no calificadas para ello (Caso \& Calonge, 2001). Juárez (1993), menciona que para que una profesión sea oficialmente reconocida y aceptada debe reunir por lo menos tres condiciones: tener un reconocimiento oficial del campo de su saber que pueda ser transmitida y certificada, contar con un área de actividad definida y legitimada por la comunidad científica y, tener un código ético que regule el ejercicio de la profesión.

Sobre la problemática del escaso reconocimiento social de las profesiones híbridas, De Ibarrola (2007), afirma que el sistema escolar ha orientado a 
los jóvenes a nuevos tipos de profesiones, no obstante, esta intención de cambio no ha sido el resultado de un desarrollo armónico entre el sistema educativo y el productivo, sino un producto de decisiones políticas derivadas de recomendaciones de organismos internacionales para superar los atrasos de nuestro país. Lo anterior evidencia una marcada contradicción entre el contexto internacional y el local, entre la globalización y la situación del desarrollo local de nuestro país, sin embargo, a pesar de lo anterior en nuestro país se siguen diseñando y ofertando carreras universitarias híbridas sin tomar en cuenta las necesidades muy particulares de cada región en la que se insertan las universidades (Planas, 2014), así los tres grandes subsistemas de educación superior mencionados al principio de este trabajo ofertan programas híbridos en todos sus planteles sin importar que se ubiquen en zonas urbanas e industrializadas o en zonas rurales con un incipiente o nulo desarrollo de tejido empresarial.

\section{La Licenciatura en Ciencias Empresariales (LCE).}

Para contextualizar la problemática estudiada se presenta un esbozo histórico del programa educativo que cursaron los egresados que participaron en la investigación. La LCE tiene sus antecedentes en la Universidad Tecnológica de la Mixteca (UTM) ubicada en la ciudad de Huajuapan de León, Oaxaca, México, pues ésta fue la primera universidad que ofertó dicha carrera en el año 1994 con el objetivo de formar profesionistas de alto nivel para su incorporación a los procesos productivos (Paz \& Herrera, 2010). En 1998 se ofertó en la Universidad de la Sierra Sur (UNSIS) localizada en la ciudad de Mihuatlán de Porfirio Díaz, Oaxaca; en el año 2002 se ofertó en la Universidad del Istmo (UNISTMO) campus Ixtepec, Oaxaca y; para agosto del año 2005 se oferta en la Universidad del Papaloapan (UNPA) campus Tuxtepec, Oaxaca. Las cuatro universidades forman parte del Sistema de Universidades Estatales de Oaxaca (SUNEO), proyecto educativo cuyo objetivo es ofrecer educación superior a los grupos de jóvenes con marcadas 
desventajas socioeconómicas para lo cual las instituciones se ubican en zonas geográficas en las que puedan captar a este tipo de jóvenes ${ }^{5}$.

El plan de estudios vigente de la carrera fue elaborado en la UTM y trasladado de manera íntegro hacia las otras tres universidades sin tomar en cuenta las características propias de cada región; no existe información sobre el grupo de especialistas que efectuó el diseño curricular, de las razones para su denominación, ni que haya respondido a los resultados de un estudio de viabilidad (Damián, 2015), es decir, en la práctica la UTM funge como el órgano rector desde el cual se dictan verticalmente las directrices y los cambios que se llevarán a cabo en la currícula del programa educativo mismas que tienen que acatar las otras instituciones del sistema. En cuanto al perfil profesional y campo de actuación del LCE, atendiendo a las características de una formación universitaria híbrida resulta ser polivalente y multidisciplinario, pues se pretende que al término de su formación universitaria el graduado incursione en una amplia gama de sectores laborales o hacerlo de manera independiente, tal como se describe a continuación:

Perfil de egreso: El LCE será un profesional de la administración científica para su incorporación en los procesos productivos del estado y de la región, será un profesional satisfecho en sus necesidades de superación personal e interesado en la investigación, cubrirá integralmente las necesidades que se generen en la actividad empresarial. [Cursivas nuestras que muestran implícitamente la formación híbrida]. Su principal actividad es en el sector privado en áreas específicas tales como administración, finanzas, compras, mercadotecnia, relaciones industriales, logística y planeación del sector industrial y comercial. También tiene amplias perspectivas laborales en las empresas de participación estatal y cooperativas, puede desempeñarse como agente independiente para la promoción y consultoría sobre organización, dirección y control. 
Campo de acción: El LCE tiene amplias oportunidades de desarrollo profesional en organizaciones públicas y privadas, nacionales o extranjeras; desempeñándose en niveles gerenciales y tomadores de decisiones en áreas como administración, finanzas, mercadotecnia, recursos humanos y logística; o de manera independiente como asesor o consultor en temas como organización y dirección de empresa, desarrollo y evaluación de proyectos de inversión, entre otros.

Sintetizando este apartado de marco teórico se puede decir que todo parece indicar que la LCE es la respuesta a las exigencias de formación profesional que demandan las organizaciones, de acuerdo a las nuevas formas de organización del trabajo que privilegian la multidisciplinariedad en lugar de la especialización. Así, de acuerdo al perfil de egreso (que va más allá de la especialización) y al campo de actuación (que carece de una delimitación específica), se espera que el egresado atienda a las diversas necesidades y exigencias del ámbito empresarial por lo cual se hace hincapié en que su desempeño laboral será en cuatro áreas específicas a saber, administración, finanzas, mercadotecnia y recursos humanos (relaciones industriales), siendo fácil identificar el proceso de hibridación de la LCE pues las cuatro áreas que se han integrado en este programa corresponden de manera independiente a carreras universitarias que por tradición y durante décadas se han ofertado en la mayoría de las IES en México bajo las denominaciones de Licenciatura en Administración, Licenciatura en Finanzas, Licenciatura en Mercadotecnia y Licenciatura en Relaciones Industriales, que tienen un perfil de egreso propio y un campo de acción delimitado, que brindan una formación específica, técnica o especializada, situación que se pretende superar con la fusión de éstas dando lugar a una formación universitaria híbrida como lo es la LCE, en la cual difícilmente el egresado puede desarrollar una identidad profesional que lo haga sentir parte de una comunidad de profesionistas y que ocasiona un escaso conocimiento social de la profesión por el conjunto de empleadores. 


\section{Método}

La investigación fue exploratoria y descriptiva con un enfoque cualitativo, se trabajó con la fenomenología pues ésta estudia a un fenómeno tal y como es percibido, experimentado y vivido por una persona (Hursserl, 1986; Creswell, 1998), aporta la inter-subjetividad y la intuición para comprender fenómenos socioeducativos y describir la realidad vivida por otros (Gurdián, 2007; Patton, 2002). Se utilizó el estudio de caso ya que nuestro interés fue una situación particular, conocer el detalle de la interacción del caso con sus contextos (Stake, 2010), y la complejidad del proceso de inserción laboral de los graduados de la LCE como profesionales híbridos para obtener evidencias y comprender las situaciones de discriminación que vivieron en dicho proceso.

La población estudiada fueron las dos primeras generaciones de egresados de la LCE, pues al ser los primeros egresados con una formación universitaria híbrida en la zona de influencia de la universidad en querer incursionar al mercado laboral, se consideró que enfrentarían mayores retos y dificultades para su inserción en el trabajo a pesar del prestigio de la universidad, pues de acuerdo a un estudio de Ranking Web de las 68 IES públicas y privadas establecidas en el estado de Oaxaca, la Universidad del Papaloapan (UNPA) ocupa el $4^{\circ}$ lugar, tomando en cuenta cuatro criterios de evaluación: presencia, apertura, excelencia e, impacto (Arellano \& Solar, 2016). La muestra no fue estadística pues la investigación cualitativa y el estudio de casos no persiguen la generalización, sino la particularización y comprender el caso a profundidad. Los criterios de inclusión para la selección de egresados fueron dos: el nivel de inserción laboral (incorporación pronta y tardía) y su trayectoria laboral (número de empleos desempeñados). A través de la técnica de muestreo "bola de nieve" se fueron agregando a los informantes iniciales claves otros informantes deteniendo el muestreo según el principio de saturación; algunos egresados se negaron a participar en el estudio mencionando que "estaban desilusionados de la Universidad y que no querían saber nada de ella", estos comentarios y su negativa en proporcionar 
información en lugar de representar un obstáculo para la investigación se constituyó en una evidencia fehaciente de una problemática que merecía ser investigada. Las características más representativas de los diez estudiantes que participaron en la investigación se muestran en la tabla 1. Cabe aclarar que tres de diez estudiantes procedían del nivel socioeconómico medio y el resto de estratos bajos; de los cinco titulados, cuatro lo hizo en un promedio de tiempo de año y medio después de concluir la formación universitaria y uno en dos años.

\section{Tabla 1.}

Datos generales de los egresados que participaron en el estudio.

\begin{tabular}{|c|c|c|c|c|}
\hline Nombre* & Título & $\begin{array}{l}\text { Trayectoria } \\
\text { laboral }\end{array}$ & $\begin{array}{c}\text { Trabajo } \\
\text { actual }\end{array}$ & Razón para estudiar LCE \\
\hline $\begin{array}{c}\text { María } \\
\text { (MA1GE1) }\end{array}$ & No & 2 empleos & $\mathrm{Si}$ & $\begin{array}{l}\text { Por problemas económicos y la LCE fue la } \\
\text { única opción. }\end{array}$ \\
\hline Rosa (RO2GE2) & $\mathrm{Si}$ & 2 empleos & $\mathrm{Si}$ & $\begin{array}{l}\text { El nombre sonaba "padre" y diferente a las } \\
\text { que ella conocía. }\end{array}$ \\
\hline $\begin{array}{l}\text { Tamara } \\
\text { (TA1GE3) }\end{array}$ & No & 2 empleos & $\mathrm{Si}$ & $\begin{array}{l}\text { Por cercanía a casa y los padres no } \\
\text { permitieron que fuera a otra ciudad. }\end{array}$ \\
\hline $\begin{array}{l}\text { Lourdes } \\
\text { (LO1GE4) }\end{array}$ & $\mathrm{Si}$ & 3 empleos & $\mathrm{Si}$ & $\begin{array}{l}\text { Padres querían "que me moviera en el } \\
\text { mundo de los negocios". }\end{array}$ \\
\hline $\begin{array}{l}\text { Amanda } \\
\text { (AM1GE5) }\end{array}$ & No & 1 empleo & $\mathrm{Si}$ & $\begin{array}{l}\text { Única opción diferente aparte del } \\
\text { Tecnológico de Tuxtepec. }\end{array}$ \\
\hline Jonás (JO2GE6) & $\mathrm{Si}$ & 3 empleos & No & $\begin{array}{l}\text { Cerca de casa y recomendación de conocidos } \\
\text { que estudiaban aquí. }\end{array}$ \\
\hline Gina (GI1GE7) & No & 1 empleo & $\mathrm{Si}$ & $\begin{array}{l}\text { Otra opción aparte del Tecnológico y porque } \\
\text { era "una carrera nueva". }\end{array}$ \\
\hline $\begin{array}{l}\text { Antony } \\
\text { (AN2GE8) }\end{array}$ & $\mathrm{Si}$ & 1 empleo & No & $\begin{array}{l}\text { No quería al Tecnológico y le llamó la } \\
\text { atención el nombre de la carrera. }\end{array}$ \\
\hline $\begin{array}{l}\text { Leonel } \\
\text { (LE1GE9) }\end{array}$ & No & Ninguno & No & $\begin{array}{l}\text { Quería estudiar Ingeniería } \\
\text { Arquitectura, por escasos } \\
\text { económicos optó por la LCE. }\end{array}$ \\
\hline Jerry (JE2GE10) & $\mathrm{Si}$ & 2 empleos & $\mathrm{Si}$ & $\begin{array}{l}\text { Quería estudiar una carrera diferente a las } \\
\text { que ofrecían otras escuelas. }\end{array}$ \\
\hline
\end{tabular}

*Atendiendo al criterio de confidencialidad se cambiaron los nombres verdaderos.

Fuente: elaboración propia con datos obtenidos en las entrevistas a los egresados participantes. 
Según la revisión de la literatura sobre el tema se diseñó una guía de entrevista que incluyó cinco variables con sus dimensiones correspondientes: trabajo previo al egreso, búsqueda del primer empleo, características del primer empleo, empleo actual y formación híbrida. Utilizando la guía correspondiente se entrevistaron a profundidad a los 10 egresados quienes proporcionaron información para analizar y describir su inserción laboral como profesionistas universitarios híbridos. Para la obtención de la información se solicitó inicialmente la participación de dos egresados mismos que después recomendaron a otros compañeros que consideraron que quizás podrían colaborar con el estudio; el contexto del desarrollo de las entrevistas fue variado pues en algunos casos se llevaron a cabo en las instalaciones de la universidad (cubículos de profesores) aprovechando la ocasión en que los egresados acudieron para solicitar informes sobre su título universitario, en los centros de trabajo de los egresados y en otros tantos en lugares públicos como cafeterías, restaurantes y bibliotecas; en todos los casos las entrevistas fueron grabadas en un sistema de audio y posteriormente fueron transcritas para su respectivo análisis y obtención de las diversas categorías.

Para el análisis de la información se utilizó el análisis de contenido pues dada la cantidad de datos obtenidos en las entrevistas esta técnica resultó adecuada al ser un "procedimiento para la categorización de datos verbales o de conducta, con fines de clasificación, resumen y tabulación" (Fox, 1981, p. 709). A fin de que el análisis de contenido permitiera una riqueza de interpretación de la información se llevó tanto a nivel manifiesto como a nivel latente (Espín, 2002), es decir primeramente se hizo referencia a los significados literales de lo que los egresados dijeron utilizando la transcripción directa de sus respuestas en función de un código determinado limitándonos a la clasificación y recuento de los términos (categorías) empleados y; en segundo lugar se intentó ir más lejos de lo dicho literalmente procurando reconstruir lo que el egresado dijo mediante la acción de llevar a cabo una inferencia de los resultados obtenidos (Álvarez-Gayou, 2005; Bogdan \& Biklen, 1992). Mediante un lenguaje sencillo se describió el proceso de 
inserción laboral en forma comprensible, con narraciones y descripciones amplias procurando no ser tan técnicas dando la oportunidad a los lectores de hacer sus propias interpretaciones del caso.

\section{Resultados}

A continuación se presentan los resultados más importantes que se obtuvieron organizados en tres apartados, primero se describen las situaciones que vivieron los egresados en las entrevistas para obtener el primer empleo, enseguida sus opiniones después de la entrevista acerca del papel que desempeñó su carrera híbrida en la consecución del empleo y, por último la situación del LCE respecto a los Contadores y Administradores en el campo de trabajo.

\section{Durante la entrevista para conseguir el primer empleo.}

Según la información proporcionada sobre las situaciones que vivieron durante la entrevista para conseguir el primer empleo, surgieron dos categorías que describen las dificultades o las formas de competencia desleal que afrontaron los egresados de la LCE con los egresados de carreras universitarias tradicionales.

\section{Categoría 1. Falta de conocimiento social de la profesión.}

Ya se explicó que el reconocimiento social de una profesión es la valoración que la sociedad le otorga a ésta o el reconocimiento por la importancia del servicio prestado por los profesionales (Bobadilla \& Torres 2002; Casado \& Calonge, 2001) y, que sea una profesión oficialmente reconocida y aceptada en sus objetivos, funciones, actividades, tareas y áreas con identidad propia (Juárez, 1993). Por lo tanto existe un correlato entre el nivel de reconocimiento social de una profesión con la demanda del profesional respectivo, así en la medida que una profesión resulte desconocida para los empleadores entonces su demanda en el mercado de trabajo será nula o escasa. Por lo 
anterior, esta primera categoría representó un factor de competencia desleal o de discriminación para la inserción laboral de los egresados según su propio imaginario, pues al no encontrar ofertas de trabajo donde solicitaran específicamente a LCE tuvieron que acudir a entrevistas emitidas para egresados de carreras tradicionales y posicionadas en el imaginario colectivo de los empleadores de las empresas locales, así María comentó:

\footnotetext{
"me entrevistaron, todo muy bien, sólo que el licenciado me comentó que ellos preferían para el puesto a una persona que hubiera estudiado Contaduría, me hizo preguntas sobre cuántas materias de contabilidad había llevado en la carrera y sobre otras materias.., a la semana me llamaron y conseguí el trabajo.., sin embargo todos los compañeros me decían contadora, yo al principio les decía: no soy contadora, pero siempre fui para ellos la contadora (MA1GE1)".
}

La situación anterior es corroborada por Antony quien se enfrentó al obstáculo de competir por un puesto de trabajo que desde el punto de vista del empleador correspondía a un egresado de la Licenciatura en Contaduría, sin embargo, menciona que por razones que desconoce fue elegido para cubrir la vacante:

"yo fui al despacho contable porque éste había solicitado personal mediante el Servicio Estatal de Empleo, así que en la entrevista me fue bien sólo que me mencionaron que ellos querían a alguien de la carrera de Contabilidad para que fuera más rápida la capacitación, pero que me darían la oportunidad y que me llamarían después.., me llamaron para empezar a trabajar al tercer día (AN2GE8)".

En los dos casos anteriores los egresados refieren que su carrera universitaria híbrida era desconocida para los empleadores originando que tuvieran que competir de manera desigual con otros candidatos al puesto que ostentaban credenciales universitarias muy conocidas en las empresas. Así, otros enfrentaron serios cuestionamientos sobre su carrera universitaria que en nada contribuyó para poder obtener prontamente el primer empleo: 
"no he conseguido un trabajo a la fecha pero a las dos entrevistas que fui casi siempre ha sido lo mismo, conocen a la UNPA pero no saben nada de la carrera de empresariales, así que te hacen muchas preguntas y pues eso ha sido difícil para mí (LE1GE9)”.

A la falta de conocimiento social de la profesión se añade la falta de identidad profesional de los egresados por lo que éstos de manera limitada tratan de "vender y convencer" a los empleadores acerca de la utilidad de su formación profesional, es decir, en la práctica la universidad ha trasladado a los egresados la responsabilidad de dar a conocer la pertinencia de su formación profesional a las empresas de la región tal como ellos mismos lo refirieron:

"casi siempre me decían o me preguntaban en la entrevista: ¿qué es esa carrera?, ¿en qué consiste?, los que nos entrevistan no saben dónde ubicarnos o si realmente vamos a poder con el trabajo, entonces tenemos que convencerlos que empresariales si nos preparó para hacer trabajos administrativos, contables, financieros y de ventas (TA1GE3)".

Igual situación de discriminación laboral por la falta de conocimiento social del LCE vivió Lourdes, quien en la etapa final de la entrevista de selección compitió con dos candidatos: un Licenciado en Economía y otro de Contaduría, por lo que ella dijo que se sintió muy abrumada y cuestionada por el tipo de formación que ostentaba a diferencia de los otros dos candidatos a quienes nunca les preguntaron acerca de su carrera universitaria:

"cuando me entrevistó la jefa de recursos humanos me comentó que había leído la solicitud de empleo y mi CV pero que tenía que hacerme algunas preguntas, total me "bombardeó" con muchas preguntas sobre la carrera, ¿en qué consistía la carrera de Ciencias Empresariales?, ¿qué temas o materias fueron los más fuertes en la carrera?, ¿si habían sido administración, contabilidad, finanzas? Me dijo: "discúlpame pero es que es la primera vez que escucho de esta carrera y no me otra queda claro para que los preparan", así que allí me tiene explicándole sobre Ciencias Empresariales (LO1GE4)". 
Llama la atención que en algunos casos los egresados de manera directa señalaron que el tener una carrera universitaria desconocida para los empleadores ocasionó que no hayan sido contratados, tal como ocurrió con Jonás quién buscó trabajo en la ciudad de Veracruz quien comentó:

"fue en TELMEX en la cual solicitaban personal para áreas administrativas, allí empecé a enfrentarme con las dificultades, recuerdo que en la entrevista la señorita de recursos humanos me atendió muy amable, todo iba bien hasta que leyó la solicitud y me dijo: "dices que estudiaste Ciencias Empresariales, ¿y qué es eso? Yo nunca he oído hablar de esa carrera”, así que medianamente le expliqué que es una carrera muy similar a Administración de Empresas y que casi podemos hacer lo mismo que ellos, total que la chica tomaba notas y terminó diciéndome que estaba bien, pero que el requisito que no cumplía era estar titulado, pero creo que eso lo dijo como una salida pues considero que al no conocer la carrera eso le dificultó el proceso de selección (JO2GE6)".

El competir de manera desigual con egresados de carreras tradicionales es una situación que se repite y no se limita solo a las experiencias de aquellos como Lourdes y Jonás que buscaron trabajo en ciudades fuera del estado de Oaxaca, sino también en aquellos que buscaron el primer trabajo en las empresas ubicadas en la ciudad donde está establecida la universidad, tal como lo expresó Amanda quien acudió a una entrevista a una tienda de autoservicios:

"fue en Chedraui, necesitaban auxiliar de recursos humanos y fui a la entrevista aunque el perfil solicitado era de Licenciatura en Administración o áreas afines, éramos como 20 candidatos. Al licenciado le sorprendió la carrera de empresariales pues él no era de aquí, me preguntó si esa carrera tenía tiempo y yo le expliqué que no, que de hecho yo era de la primera generación, entonces me preguntó sobre los temas de RH que había visto en la carrera, me dijo que evaluaría mi solicitud y que si les interesaba me llamarían, cosa que nunca sucedió... la verdad me sentía muy mal, triste (AM1GE5)”. 
La competencia desleal por los puestos de trabajo y la discriminación del egresado de la LCE con otros profesionistas por la falta de conocimiento social de la carrera originó que en algunos casos a pesar de que los empleadores no conocían la carrera de Ciencias Empresariales, éstos decidieran darles la oportunidad a los egresados para incorporarse a la empresa a trabajar a fin de "ponerlos a prueba" y "saber que podían hacer" o como se desempeñaban en el trabajo asignado, tal como ocurrió a Gina quien comentó:

"en COMPARTAMOS me trataron bien.., me comentó la persona de recursos humanos que yo era la primera con la carrera de empresariales que contrataban y que estarían al pendiente de mi desempeño para futuras contrataciones, después de un año me di cuenta que en otra sucursal habían contratado a una egresada de la segunda generación por lo cual creo que si me he desempeñado bien (GI1GE7)".

En otros casos como el de Jerry, los entrevistados atribuyen el haber conseguido su primer empleo debido al prestigio que se ha ganado la universidad en la ciudad por su sistema de trabajo muy exigente y no porque los empleadores conocieran su carrera universitaria, así el comentó:

"a mí siempre me han tratado bien a pesar de que coincido con la mayoría de los compañeros de que igual me hacen preguntas sobre la carrera, pues no es conocida a diferencia de los Contadores y Administradores [egresados] del Tecnológico (JE2GE10)”.

En el caso de Rosa no se presentaron dificultades para encontrar el primer empleo pues se incorporó a una empresa propiedad de un familiar, sin embargo, mencionó que el administrador general de dicha empresa tenía muchas dudas sobre su perfil profesional y por ende para asignarle actividades o funciones que pudiera desarrollar:

“... a pesar de que el dueño del negocio es mi tío, el me presentó con un señor que es algo así como su administrador y le dijo que me explicará sobre el trabajo, así que prácticamente no hubo una entrevista para conseguir el empleo más bien el objetivo de esta presentación era que 
el administrador quería saber que había estudiado, que sabía hacer, etc. para asignarme los trabajos o ayudarle en cosas que él estaba realizando (RO2GE2)".

\section{Categoría 2. Falta de Identidad profesional.}

La identidad profesional implica pertenecer a cierto grupo, identificarse como profesionista en un campo delimitado o específico, con un alto nivel de habilitación profesional, gozar del respaldo y reconocimiento institucional y, tener una idea clara y definida de lo que implica pertenecer a esa profesión (Ávila \& Cortés, 2007; Hatum \& Rivarola, 2007). El egresado universitario a lo largo de su formación desarrolla una identidad profesional que le otorga cierto nivel de seguridad y confianza en las entrevistas de trabajo pues conoce la delimitación de su actuar profesional y puede dar razones sobre las áreas de la empresa en donde puede desempeñarse. No obstante, esta segunda categoría contribuyó -según el imaginario de los entrevistados-, en cierta medida a la discriminación y a obstaculizar su pronta inserción laboral pues cuando los empleadores les cuestionaban acerca de su carrera universitaria, del perfil profesional y las funciones que podrían realizar, éstos escasamente pudieron dar razón o defensa de la misma, coincidiendo con la teoría que afirma que los profesionales híbridos escasamente desarrollan una identidad profesional y tienen problemas para delimitar su actuación profesional, lo anterior es corroborado por los entrevistados quienes mencionaron:

\footnotetext{
"los que estudiamos Ciencias Empresariales tenemos problemas para saber exactamente a que dedicarnos en comparación con otros.., por ejemplo, mi primer trabajo fue en una pequeña empresa editorial como auxiliar contable, aunque sé que no soy contadora pero allí la llevamos (TA1GE3)".
}

En otros casos los egresados reconocen que al ser cuestionados por los empleadores en las entrevistas de trabajo acerca de su carrera híbrida, no fueron capaces de explicar o hicieron a medias una "buena defensa" de 
la misma y que nunca imaginaron que tuvieran que enfrentarse con esta problemática que los coloca en desventaja en comparación con egresados de carreras ya posicionadas en el conjunto de empleadores:

\footnotetext{
".., casi en todas las entrevistas a las que fui siempre me preguntaban sobre la carrera y yo "a medio explicar" en qué consiste y digo a medio explicar porque es la verdad, yo no iba preparado para ello, nunca pensé que no conocieran la carrera de empresariales y que yo tuviera que "venderla" o convencer de lo buena que es la carrera en las entrevistas (JO2GE6)".
}

\section{Después de la entrevista para conseguir el primer empleo.}

Concluida la entrevista de trabajo y según el resultado de la misma los egresados opinaron sobre el papel que desempeñó su formación universitaria híbrida para su inserción laboral. Al igual que en el apartado anterior los entrevistados hacen referencia constante al desconocimiento de su carrera universitaria híbrida en las empresas como un factor negativo y como un factor de discriminación para poder encontrar el primer empleo, tal como se describe a continuación.

\section{Categoría: Falta de conocimiento social de la profesión.}

Esta categoría corroboró la falta de conocimiento social de la LCE para los empleadores quienes al no tener una delimitación clara de la actuación profesional de los egresados tuvieron confusión y dificultades para identificar los diversos trabajos que pueden llevar a cabo y por ende para ofrecerles puestos de trabajo. Reiteradamente los egresados refieren que la falta de conocimiento social los coloca en desventaja en comparación con los egresados de carreras universitarias que gozan de un amplio reconocimiento de los empleadores: 
"en muchas ocasiones me preguntaban: ¿bueno que es lo sabe hacer alguien que estudió tu carrera? ¿en qué son buenos?, y es difícil estar diciendo que sabemos de todo un poco, pues yo notaba que la persona que nos entrevistaba no lo convencía esa respuesta, me daba la impresión que imaginaba que yo no tenía bien claro en qué tipo de trabajo era buena (MA1GE1)".

El desconocimiento de la carrera por parte de los empleadores causó desánimo en algunos entrevistados a pesar de que en un principio se mostraban animosos, situación que resulta grave ya que los teóricos afirman que el tener una actitud positiva es un factor individual de mucho peso para afrontar con éxito el proceso de búsqueda del primer empleo; la situación anterior bien lo describió Tamara:

"es un poco complicado opinar sobre esto pues hay de todo... ¡asu! [Expresión coloquial en la región] como explicarlo, por ejemplo la carrera es buena, nos preparan bien, entonces uno va con ganas a las entrevistas y izas! te encuentras con una barrera: no conocen que es un Licenciado en Ciencias Empresariales y pues eso te desmotiva, te sientes como un ser raro en comparación con otras personas que tienen carreras muy conocidas (TA1GE3)".

De manera implícita la mayoría de los entrevistados hacen suyo uno de los objetivos de las carrera híbridas al afirmar que su carrera es buena por la formación multidisciplinaria que recibieron $\mathrm{y}$, que por lo tanto debería facilitarles conseguir un empleo, sin embargo, consideran que el desconocimiento de la misma por los empleadores origina una discriminación o una competencia desleal por los puestos de trabajo dificultando su rápida inserción laboral:

"la carrera debería de facilitar conseguir trabajo debido a que nos enseñan muchas cosas, pero como no conocen a la carrera en las empresas allí está el problema, creo que si la conocieran rápidamente nos colocaríamos pues podemos estar un muchas áreas o departamentos, pero siempre en las entrevistas me tenían que decir que explicara sobre ella, era como un interrogatorio a diferencia de 
otros egresados de otras carreras que luego saben para que los van a contratar (LE1GE9)".

El problema de la falta de conocimiento social del LCE no solo ocurre en la ciudad donde se ubica la universidad sino también en otras ciudades del estado de Oaxaca a donde algunos egresados viajaron con el objetivo de conseguir el primer empleo con mejores condiciones de trabajo, tal como fue el caso de Lourdes quien comentó:

"la carrera es buena y nos capacita para muchas cosas, sin embargo es el desconocimiento que tienen [los empleadores] de ella lo que dificulta encontrar un empleo, a mí me llama la atención que en la capital, en Oaxaca, no conocen la carrera y eso que la UTM [universidad del sistema] tiene más de 15 años de ofrecerla y que nos digan que la carrera de Ciencias Empresariales es la que ha tenido los mejores lugares [CENEVAL] a nivel nacional, entonces, como no conocen la carrera en las entrevistas te cuestionan o preguntan de ella, así que si eres desenvuelto sabes explicar bien en que consiste, pero si eres tímido o estas nervioso el asunto empeora y los empresarios dirán que ni siquiera uno que estudió esa carrera sabe para qué somos buenos (LO1GE4)".

Lourdes muestra la necesidad de que los egresados desarrollen una identidad profesional a fin de que puedan "dar cuenta o vender" con éxito a los empleadores sus conocimientos, habilidades y destrezas que adquirieron como profesionales híbridos, pues cuando no lo pueden hacer, desde su percepción, se constituye en un obstáculo para su inserción laboral tal como lo comentó Amanda quien reconoció su incapacidad para explicar su perfil profesional:

"el no saber exactamente a que se dirige la carrera fue una barrera $u$ obstáculo, muchas veces en las entrevistas cuando me preguntaban qué puede hacer un Licenciado en Ciencias Empresariales yo siempre decía que "de todo un poquito" y notaba que quién me hacía la entrevista no se convencía con la respuesta, me daba la impresión que se imagina que ni yo sabía a qué podía dedicarme en específico 
o en qué área era mejor y, no andaban tan equivocados pues es la verdad, en la carrera nos enseñan muchas cosas para desempeñar bien el trabajo, pero que en nuestro caso eso precisamente representa una debilidad para encontrar un empleo (AM1GE5)".

En otros casos los egresados refieren que por ser de las primeras generaciones no son conocidos y que por lo mismo enfrentan una discriminación muy marcada en el campo laboral en el cual los empleadores prefieren optar por opciones ya probadas, es decir, contratando en primer lugar a los egresados de carreras muy posicionadas en el imaginario colectivo de los mismos:

"la carrera de empresariales pues tanto te ayuda como también dificulta encontrar empleo, yo creo que con el correr del tiempo quizás todo sea más favorable, pero como todavía somos nuevos, de las primeras generaciones entonces en las empresas no nos conocen y eso resta puntos en las entrevistas, aunado a que nunca solicitan a alguien de Ciencias Empresariales, entonces ya desde el principio hay una separación o discriminación hacia nosotros (JO2GE6)”.

En la misma tónica algunos entrevistados directamente "señalan, acusan" o atribuyen el no poder insertarse fácilmente al campo laboral por el desconocimiento que tienen los empleadores de su carrera universitaria hibrida y, que tienen que andar "rogando" para que les den la oportunidad de demostrar su capacidad para el trabajo, situación que les provoca descontento como se muestrea en el siguiente comentario:

"pues claro que con la carrera se dificulta encontrar trabajo, esto a pesar que estamos muy bien preparados y tenemos una formación en muchas áreas, pero eso de que nos sirve pues sólo nosotros lo sabemos y en las empresas no, al parecer siempre prefieren a las opciones ya conocidas y probadas y no nos queda de otra que casi estar pidiendo la oportunidad de demostrarles que podemos hacer el trabajo (AN2GE8)". 
Tan desconocida es la carrera de LCE en la zona de influencia de la universidad que sobre este tema Gina comentó que durante un año de búsqueda para encontrar el primer empleo nunca vio o se enteró de una oferta de trabajo donde solicitaran a alguien con su carrera universitaria, así que se vio obligada a tener que ir a entrevistas de trabajo que eran para egresados de carreras afines, denotándose la competencia desleal y la discriminación en el campo de trabajo:

"la mera verdad la carrera no te ayuda mucho para conseguir empleo, yo en más de un año de leer casi a diario las ofertas de trabajo en el periódico nunca, pero nunca encontré que solicitaran a un Licenciado en Ciencias Empresariales, siempre pedían a egresados de Administración y Contadores y por último decían o de áreas afines, donde creo que entramos nosotros, pero eso es ya lo último, lo que quiere decir que si no hay administradores y contadores pues ya ni modo que sean de empresariales, entonces nuestra carrera no es conocida y no nos toman en cuenta en las ofertas de trabajo (GI1GE7)".

Ante esta situación algunos entrevistados consideran que es necesario revertir esta situación mediante dos acciones, aprovechando el prestigio que está ganando la universidad por la exigencia de su sistema educativo y, a través del buen desempeño en el trabajo de aquellos egresados que ya están insertado en el mercado de trabajo tal como lo sugiere Jerry:

\footnotetext{
"yo reconozco que la mayoría ha tenido problemas [para encontrar trabajo] por ser una carrera nueva o desconocida, pero nosotros debemos poner de nuestra parte y poco a poco revertir esto, por ejemplo, en mi caso cuando fui a la entrevista el hecho de que la cervecera haya solicitado a estudiantes o egresados de la carrera habla bien pues lo que ellos saben es la buena preparación de la UNPA y eso se debe aprovechar, así que cuando nos contraten tenemos que hacer un buen trabajo, desempeñarnos bien y con eso nosotros abriremos las puertas para que otros compañeros sean aceptados rápidamente (JE2GE10)".
} 


\section{La lucha en la "arena profesional": el LCE frente a otros profesionistas del área económico administrativa.}

Para mostrar la posición desigual o desventajosa que guardan los egresados de la LCE en el campo laboral frente a los Contadores y Administradores, a continuación se describen las percepciones de los mismos ${ }^{6}$. Todos los entrevistados refieren que ningún empleador y/o responsable de Recursos Humanos tenían conocimiento de la existencia de la carrera y por ende de sus egresados, representando un obstáculo para su rápida inserción laboral pues los empleadores generalmente contratan a profesionales de carreras económico administrativas tradicionales tal como lo expresó María:

\footnotetext{
"los propietarios de las empresas o los responsables de RH no saben de la carrera de empresariales ni de su tipo de formación, yo creo que por eso nunca he visto en los periódicos que soliciten a Licenciados en Ciencias Empresariales y eso es una barrera para encontrar un empleo, ellos contratan a contadores y administradores pues son carreras muy antiguas y que además el Tecnológico siempre ha ofrecido (MA1GE1)".
}

Ante lo anterior algunos entrevistados como Jonás, refieren que a pesar de que pueden llevar a cabo diversas actividades del área contable debido a su formación universitaria generalista están en desventaja, pues los empleadores rápidamente ubican en éstas áreas a los egresados de Contaduría presentándose una discriminación laboral hacia ellos:

"la mayoría no sabe de nosotros, sino no nos preguntaran tanto en las entrevistas acerca de nuestra carrera y para que nos prepararon en la universidad, eso incluye que igual no sepan de nuestro perfil profesional, yo puedo ir a solicitar el puesto de auxiliar contable o contralor pues sé que mi carrera me capacitó para ello, pero al

6 La posición desigual y de discriminación del egresado de la LCE frente a otros profesionistas de áreas afines se refiere al proceso de inserción laboral, pues de acuerdo a resultados preliminares de una investigación en curso una vez que éstos logran insertarse en su primer empleo, no se observan diferencias en cuanto a las actividades laborales desarrolladas, nivel ocupado (auxiliares administrativos) y salario percibido. 
empleador le sorprende pues ya tiene en la mente que eso corresponde al perfil de un Licenciado en Contaduría y no del [Licenciado en] Ciencias Empresariales por lo que eso nos pone en desventaja (JO2GE6)".

Tal es el desconocimiento de la LCE que en la propia ciudad donde está ubicada la universidad los empleadores creen que los egresados que ostentan este título universitario se formaron en IES de otros estados de la república $y$, exigen a los egresados que expliquen o den razones por las cuales creen que pueden efectuar trabajos relacionados con el área de contabilidad, la situación anterior fue bien ilustrada por Tamara:

"el conocimiento es nulo pues en mi primer trabajo no conocían a la carrera ni a que se dedica uno de empresariales, luego me dijeron que si yo no era de contabilidad porque creía que podía hacer el trabajo, así que tuve que explicar que nosotros a diferencia de los administradores llevamos más materias de contabilidad en la carrera y que consideraba que para el puesto de auxiliar contable si podría desarrollar el trabajo, igual cuando vine a la entrevista para este trabajo [actual] la persona que me entrevistó se sorprendió del nombre de la carrera y me dijo que en qué estado [de la república] había estudiado pues nunca había oído de esta carrera, así que otra vez a explicarle todo sobre ella, pero no soy la única creo que todos mis compañeros han pasado por esto de estar explicando que es un Licenciado en Ciencias Empresariales (TA1GE3)".

La falta de conocimiento social de la profesión origina que los entrevistados enfrenten un proceso de inserción laboral discriminatorio, pues los empleadores al otorgarles un puesto de trabajo lo hacen para que "demuestren" lo que pueden hacer con su formación universitaria desconocida y que estarán a prueba acerca de su desempeño tal como lo expresó Gina:

"ese es un gran problema que la mayoría hemos afrontado pues no nos conocen, aquí en COMPARTAMOS yo fui la primera que fue contratada y tuve que competir con otros que querían el puesto, pero noté que al reclutador no le convencía la carrera ya que varias veces 
me dijo que no sabía que era, que no había oído de ella, aunque al final me dijo que si era una carrera del área económico administrativa podría darme la oportunidad para demostrar que es lo que sabía hacer y que iba a estar a prueba por tres meses (GI1GE7)".

\section{Los empleadores no conocen a la LCE pero identifican a la Universidad} por ofertar una carrera con un nombre "raro" misma que les produce incertidumbre y que la asemejan a la Licenciatura en Administración, situación que los egresados consideran como una desventaja tal como lo comentaron Amanda y Rosa:

"en la mayoría de las entrevistas que tuve la carrera de [Ciencias] Empresariales no era conocida, sólo conocían o habían oído hablar de la UNPA pero eso no sólo yo lo he encontrado, pues igual comentamos con otros compañeros que también encontraron que en las empresas no conocen la carrera y ni a que se puede dedicar o donde se puede colocar a un egresado de esta carrera (AM1GE5)".

"las empresas no conocen la carrera de empresariales, mi tío siempre me decía que le explicara en qué consistía la carrera por que él nunca había oído de ella y en mi segundo empleo cuando me preguntaron si yo era de la UNPA, quien me entrevistó dijo que la escuela era la única que impartía esa carrera con un nombre raro, pero que para él era administración pero con otro nombre, entonces se ve que existen muchas dudas allá afuera sobre la carrera o lo que nosotros somos y eso es algo como una mancha [ríe], como una desventaja (RO2GE2)"

La situación no fue diferente para aquellos que buscaron trabajo en otras ciudades como fue el caso de Lourdes quien creyó que en la Ciudad de Oaxaca y en el puerto de Salina Cruz no tendría dicho problema pues son ciudades cercanas a otras universidades del SUNEO que desde hace años ofertan la $\mathrm{LCE}^{7}$, sin embargo, dicha carrera universitaria es desconocida en esas ciudades: 
"en los tres trabajos no conocían a la carrera de [Ciencias] Empresariales y eso que mis trabajos les he tenido fuera de Tuxtepec; en CEMEX y en esta consultoría en la ciudad de Oaxaca cuando llegué creí que ya sabrían de la carrera pues según la UTM [Universidad Tecnológica de la Mixteca] tiene más de 10 años de ofrecer la carrera y por su cercanía creí que tenía muchos egresados laborando aquí en la capital, pero pues no es así; igual en Salina Cruz pues está cerca UNISTMO [Universidad del Istmo] que ofrece empresariales y también la carrera no es conocida, somos desconocidos [en las empresas] y tenemos que estar explicando en las entrevistas que existimos y que sabemos hacer bien las cosas (LO1GE4)".

Abonando al comentario de Lourdes, Antony quien viajó hasta el estado de Tabasco en busca de oportunidades de trabajo refiere que en las empresas de ese estado desconocen la LCE y que al regresar a su ciudad de origen al igual que sus compañeros enfrentó dicha problemática pues los empleadores locales conocen a las carreras de Contaduría, Administración, Mercadotecnia y Dirección de Negocios ofertadas por otras universidades:

"en Villahermosa [Tabasco] fui a varias entrevistas y encontré que la carrera era desconocida pero yo pensé que era por la distancia y por ser otro estado de la República pero no fue así, cuando regresé a Tuxtepec el asunto no fue diferente pues en las empresas conocen a la UNPA pero no a la carrera y por lo tanto no saben en qué puestos específicamente nos pueden colocar a diferencia de los egresados del Tecnológico, de la UMAD [Universidad Madero] y de la UNID [Universidad Interamericana para el Desarrollo] que tienen carreras bien conocidas y reconocidas en las empresas (AN2GE8)".

Luego entonces la falta de reconocimiento social de la LCE como carrera universitaria híbrida representa un obstáculo para su inserción laboral y se constituye en un reto para ellos afín de que mediante su desempeño en las empresas puedan abrir espacios laborales para las nuevas generaciones:

por lo cual a pesar del tiempo que ha transcurrido son muy pocos los egresados que se encuentran en el campo laboral (Damián, 2017). 
"los egresados de [Ciencias] Empresariales tenemos un gran reto que consiste en abrir las puertas y los espacios en las empresas para las futuras generaciones, pues a pesar de que nuestra carrera nos prepara muy bien en las empresas somos desconocidos, no están enterados de lo que sabemos hacer ni de nuestra formación en varias áreas, lo anterior hace que efectivamente a la mayoría de los compañeros les haya costado colocarse o encontrar rápido un trabajo (JE2GE10)".

\section{Discusión y Conclusiones.}

Este acercamiento exploratorio sobre la competencia desleal y la situación discriminatoria que afrontan en el proceso de inserción laboral los egresados universitarios híbridos, aporta muchos elementos para el debate y abre interesantes líneas de investigación en este campo emergente de la investigación educativa tal como lo refieren Jiménez (2009) y Damián (2014), quienes han abordado este tema en el país. Se reconoce que mucha tinta ha corrido sobre la temática de estudios y seguimiento de egresados pero escasamente se ha indagado bajo el contexto y las exigencias de las políticas educativas actuales, acerca del impacto de la oferta y formación de nuevos cuadros profesionales en la demanda de dichos cuadros en el mercado de trabajo (Barrón y Gómez, 2004) y según Planas (2014), conocer el grado de correspondencia entre las nuevas carreras universitarias y las exigencias actuales de acuerdo a la actual forma de organización del trabajo, por lo cual es necesario atender este tema ya que la investigación educativa además de producir conocimiento científico también debe contribuir en señalar los aciertos y los desatinos de las políticas educativas actuales (Damián, 2014).

Si bien es cierto que en la literatura se alude a la existencia de varios factores para marcar, señalar y segregar a los egresados al momento de su incorporación al mercado de trabajo, tales como el estrato socioeconómico de procedencia, el sexo, la edad, el prestigio de la universidad que los formó, entre otros (ANECA, 2009), las evidencias empíricas obtenidas en este estudio de caso apuntan que las carreras universitarias híbridas están originando una nueva forma de exclusión y discriminación laboral originada por el tipo de credencialización universitaria por la que opte el individuo (Damián, 2015), 
es decir, su inicio en el mundo del trabajo estará condicionada en cierta medida por haber decidido estudiar una carrera universitaria tradicional o una de carácter multidisciplinaria o híbrida (Diamant, 2009; Díaz, 2005; Quiróz, 2014).

Las dificultades que afrontan los egresados de la LCE en el proceso de inserción laboral contradicen las ventajas atribuidas a las carreras híbridas en el sentido de facilitar la inserción en el trabajo (Holguín, 2011; Sáez, 2007); todo apunta como señala Barrón (2005) y Barrón y Gómez (20024), que dichos programas educativos preparan cuadros profesionales o recursos humanos que son "inexistentes" en la pirámide ocupacional de las organizaciones, ocasionando que los empleadores al desconocer la nueva oferta de profesionales híbridos no oferten plazas de trabajo para éstos quedando en una posición desventajosa ante los egresados que ostentan una carrera universitaria tradicional con un alto grado de aceptación o reconocimiento social.

Cuando los egresados híbridos son cuestionados sobre su tipo de formación universitaria, éstos son incapaces de dar buenas razones o hacer una buena defensa de su formación híbrida originada por la falta de identidad profesional misma que no fue desarrollada por carecer de un referente externo del profesional aun durante su formación en las aulas (Paéz, 2009; Dubar, 2000), por lo que en la búsqueda del primer empleo se presenta la situación de exclusión, señalización y de competencia desigual por las ofertas de trabajo con aquellos individuos que apostaron por opciones universitarias tradicionales y que son favorecidos en el proceso de reclutamiento y selección de personal (Diamant, 2009).

En el estudio de caso analizado la discriminación, competencia desleal y exclusión del profesional híbrido es evidente al no encontrar éste ofertas de trabajo tanto en el estado de Oaxaca, Veracruz y Tabasco donde solicitaran personal con el título de LCE en medios tanto impresos como electrónicos y, ante la necesidad de incorporarse al trabajo tuvieron que acudir a entrevistas donde solicitaban a profesionales de carreras tradicionales como opciones 
probadas a los cuales los empleadores favorecen en el proceso de contratación $\mathrm{y}$, sólo en caso de quedar desiertas dichas ofertas de trabajo les brindan la oportunidad de incorporarse de manera temporal bajo ciertas "reservas o condiciones", a fin de que demuestren lo que saben hacer en el plano laboral como individuos que ostentan una carrera universitaria desconocida.

Si bien se reconoce que este trabajo reporta resultados de un estudio de caso de carácter exploratorio y descriptivo y que es aplicable en el contexto particular o institucional donde se llevó a cabo, actualmente las IES públicas de México en su conjunto están siendo sometidas a diversas exigencias desde el ámbito gubernamental para que oferten nuevas carreras universitarias entre las cuales destacan aquellas que ofrecen una formación trans, inter y multidisciplinaria, por lo cual se puede afirmar que dichas medidas son la respuesta a la nueva política educativa nacional de educación superior (Planas, 2014; De Ibarrola, 2007; Muñoz y Rodríguez, 2004). Por lo anterior, a manera de cierre y en forma de conclusión prospectiva, para abrir el debate para los interesados en investigar esta temática bien cabría hacer tres grandes cuestionamientos a los responsables de la política educativa universitaria en el país: ¿Por qué los empleadores no conocen a las carreras híbridas y discriminan a los egresados que ostentan esta formación? ¿Acaso no se impulsan estas carreras híbridas como producto de un diagnóstico serio de las necesidades del mercado de trabajo? ¿Existirán realmente diferencias en los contenidos curriculares de las carreras hibridas con respecto a las carreras tradicionales o, se tratará sólo de una estrategia de marketing educativo otorgando nombres o denominaciones diferentes que resulten atractivas para allegarse de matrícula? Consideramos que es importante dar un seguimiento a la situación de los profesionales híbridos en el campo de trabajo generando conocimiento mediante la investigación, pues todo parece indicar que la formación universitaria híbrida lejos de contribuir a la rápida inserción en el mercado de trabajo, se constituye en un nuevo elemento de señalización o discriminación originando en la "arena profesional" una lucha o competencia 
en condiciones de desventajas con respecto a los profesionistas con una formación tradicional.

\section{Referencias}

Álvarez-Gayou, L. (2005). Cómo hacer investigación cualitativa. Fundamentos y metodología. México. Paidós.

ANECA. (2009). Los procesos de inserción laboral de los titulados universitarios en España. Factores de facilitación y obstaculización. Madrid. Agencia Nacional de Evaluación de la Calidad y Acreditación.

Arellano, J., \& Soler, R. (2016). Clasificación Web de las Instituciones de Educación Superior Públicas y Privadas del Estado de Oaxaca. Temas de Ciencia y Tecnología, 20(59), 33-42.

Ávila, J., \& Cortés, J. (2007). La construcción de las identidades profesionales a través de la educación superior. Cognición, 1(9), 52-62.

Barrón, C., \& Gómez, J. (2004). Las nuevas profesiones en las instituciones de educación superior". En Á. Valle (Coord.), Las profesiones en México frente al mercado de trabajo (pp. 47-94). México. CESU-UNAM.

Barrón, C. (2005). Formación de profesionales y política educativa en la década de los noventa. Perfiles Educativos, 37(108), 45-69.

Bobadilla, P., \& Torres, C. (2002). ¿... y si no es tu vocación? Mecanismos de elección profesional y la opción por la sociología en estudiantes de la PUCP. Avances de investigación. Debates en sociología, 27, 95-129.

Bogdan, R., \& Biklen, K. (1992). Qualitative research for education: An introduction to theory and methods. Boston: Allyn \& Bacon.

Caso, E., \& Calonge, S. (2001). Conocimiento Social y Sentido Común. Caracas: Fondo editorial de Humanidades y Educación. Universidad de Venezuela.

Creswell, W. (1998). Qualitative Inquiry and Research Design: Choosing Among Five Traditions. Thousand Oaks, California: Sage Publications, Inc.

CUP. (2009). Modelo Educativo de las Universidades Politécnicas. Recuperado de: http://politecnicas.sep.gob.mx/ModeloEducativo.html 
Damián, J. (2017). Sobreviviendo a un sistema universitario de alta exigencia: valoraciones de alumnos próximos a egresar, en A. Barraza y V. Gutiérrez (Coord.). El estudio de la realidad educativa a partir de sus actores (pp.87-115). México. Instituto Universitario Anglo Español.

Damián, J. (2015). Factores que facilitan y obstaculizan la inserción laboral de los egresados de carreras hibridas. El caso del Licenciado en Ciencias Empresariales de la Universidad del Papaloapan (Tesis inédita de doctorado). México. Centro de Investigación y Docencia en Humanidades del Estado de Morelos.

Damián, J. (2015a). Professional Identity, Social Recognition and Entering the Workforce of the University Student with Hybrid Education. Propósitos y Representaciones, 2(2), pp. 45-76. doi: 10.20511/pyr2014. $\mathrm{v} 2 \mathrm{n} 2.60$

Damián, J. (2014). La formación universitaria híbrida: Retos y oportunidades. Revista Actualidades Investigativas en Educación, 14(2), 1-22.

Dahrendorf, R. (1986). La flexibilidad del mercado de trabajo. Informes $O C D E$. Madrid. Editada en español por el MTSS.

De Ibarrola, M. (2007). Formación escolar para el trabajo: posibilidades y límites. Experiencias y enseñanzas del caso mexicano, en De Ibarrola (Ed.), Nuevas tendencias de la formación escolar para el trabajo. OIT/ Cinterfor

Diamant, A. (2009). Recorridos iniciales en la construcción de la identidad profesional de los psicólogos en la universidad de Buenos Aires de los 60's". Anuario de Investigaciones, 16, 163-172.

Díaz, M. (2005). Identidad del licenciado en nutrición de la UAQ (Tesis de Maestría). Universidad Autónoma de Querétaro. Santiago de Querétaro, México.

DGEST. (2009). Diseño e Innovación curricular para el desarrollo de competencias profesionales en el SNEST. Recuperado de http://www. dgest.gob.mx

Dubar, C. (2000). La crisis de las identidades. La interpretación de una mutación. Trad. Por José Miguel Marcen. España: Bellaterra. 
Espín, V. (2002). El análisis de contenido: una técnica para explorar y sistematizar información. Revista de Educación, 4, 95-105

Fox, D. (1981). El proceso de investigación en educación. Eunsa. Pamplona.

Gurdián, A. (2007). El Paradigma Cualitativo en la Investigación SocioEducativa. San José, Costa Rica. Coordinación Educativa y Cultural Centroamericana (CECC)-Agencia Española de Cooperación Internacional (AECI).

Hatum, A., \& Rivarola, R. (2007). La carrera profesional: navegando entre sus dilemas. Buenos Aires: Granica.

Holguin, C. (2011). Carreras híbridas, cambiando el paradigma de la especialización. Revista UNIVERCITIES, 1(4), 3-4.

Husserl, E. (1986). Ideas relativas a una fenomenología pura y una filosofía fenomenológica. México: Fondo de Cultura Económica.

Jenschke, B. (2004). Orientación para la carrera. Desafíos para el nuevo siglo bajo una perspectiva internacional I, en Memoria académica Orientación y Sociedad - 2003 - 2004 (vol.4, pp. 13-24).Universidad Nacional de la Plata,

Jiménez, M. (2009). Trayectorias laborales y movilidad de los biólogos agropecuarios de la Universidad Autónoma de Tlaxcala. Perfiles Educativos, 31(126), 56-79.

Jiménez, M. (2009a). Desarrollo profesional, laboral y socioeconómico del Biólogo Agropecuario de la Universidad Autónoma de Tlaxcala. Valoraciones de los egresados de una carrera profesional híbrida. Trabajo presentado en el $\mathrm{X}$ Congreso Nacional de Investigación Educativa, Veracruz.

Juárez, M. (1993). El trabajo social. ¿Una nueva lectura?”, en M. Juárez (Ed.), Trabajo Social e Investigación. Temas y perspectivas (pp. 17-30). Madrid, Universidad Pontificia Comillas.

Meijers, F. (1998). The development of a career identity. International Journal for the Advancement of Counselling, 20, 191-207. Netherlands.

Muñoz, H., \& Rodríguez, R. (2004). La educación superior en el contexto actual de la sociedad mexicana. Cuadernos del Seminario de Educación Superior. 
Paz, Y., \& Herrera I. (2010). Instituto de Ciencias Sociales y Humanidades. Temas de Ciencia y Tecnología, 14(40), pp.61-63

Paéz, M. (2009). Socialización e identidad profesional. Recuperado de http:// www.upn25b.edu.mx/.../Paez\%20Gutierrez\%20Maricela.pdf

Patton, Q. (2002). Qualitative research \& evaluation methods. Thousand Oaks, CA: Sage.

Planas, J. (2014). Adecuar la oferta de la educación a la demanda de trabajo, ¿es posible? Una crítica a los análisis "adecuacionistas" de relación entre formación y empleo. México. ANUIES.

Psacharopoulos, G. (2005). Linking vocational education and training research, policy and practice: a personal view. Vocational Training European Journal, 36(3), 69-73

Quiroz, M. (2014). Formación polivalente e identidad profesional de los interventores educativos. Revista de la Educación Superior, 43(4), 101121.

Sáez F. (2007). Necesitamos ingenieros sociotécnicos. Conferencia presentada en el Seminario La ingeniería ante las Ciencias Sociales y las Humanidades. Recuperado de: http://netosfera.1blogs.es/2007/12/12/ hibrídosfera/

Stake, R. (2010). Investigación con estudio de casos. Madrid: Morata

Universia. (2009). El futuro está en las carreras híbridas [Universia.net]. Recuperado de http://www.universia.net 